\title{
In vitro analysis of urinary stone composition in dual-energy computed tomography
}

\author{
Monika Stępieńn ${ }^{1 A, B, C, D, E, E, G, G}$, Robert Chrzan ${ }^{1 A, B, C, D, E}$, Wojciech Gawlas ${ }^{2 A, B, F, G}$ \\ 'Department of Radiology, Jagiellonian University in Kraków, Collegium Medicum, Poland \\ 2Department and Clinic of Urology, Jagiellonian University in Kraków, Collegium Medicum, Poland
}

\section{Abstract}

Purpose: Dual energy computed tomography (DECT) is a new method of computed tomography (CT) imaging, allowing the assessment of not only the object's morphology, but also its composition. The aim of the study was to evaluate the potential of in vitro DECT evaluation of urinary stones' chemical composition.

Material and methods: Six samples of surgically removed renal stones were scanned using DECT and analyzed by scanner vendor software. Uric acid stones were marked red and calcium stones white by the software. The real composition of the stones was finally verified using physicochemical laboratory analysis.

Results: In 5 out of 6 samples, the composition of stones in DECT ( 3 samples identified as uric acid and 2 samples as calcium) was consistent with the physicochemical analysis (3 samples identified as uric acid, 1 as calcium phosphate, 1 as calcium oxalate). In DECT it was not possible to determine more precisely the type of calcium compounds (calcium phosphate vs. calcium oxalate) as established in the physicochemical analysis.

In one stone identified in physicochemical analysis as uric acid, DECT detected a composite layered structure containing both uric acid and calcium compounds.

Conclusions: DECT allows uric acid to be distinguished from calcium urinary tract stones, which is crucial in the choice of appropriate therapy. Using the available hardware and software, it was not possible to more accurately distinguish types of calcified stones. Evaluation of the stone type in DECT may be limited in the case of mixed chemical composition.

Key words: urinary stone, dual energy computed tomography.

\section{Introduction}

Urolithiasis is a common disease associated with the presence of insoluble deposits in the urinary tract, which arise as a result of the precipitation of chemicals in the urine, when their concentration exceeds the solubility threshold. Kidney stones are found in 10-12\% of men and about 5-6\% of women throughout life [1]. Deposits can arise in various sections of the urinary tract, most commonly in the calyces and renal pelvis, and then move to the ureter or bladder where they can grow or be excreted in the urine.

Sometimes the deposits reach a considerable size, and fill the entire renal pelvis and calyces (staghorn calculi), leading to kidney damage.

The most common deposits are composed of calcium oxalate, less frequently calcium phosphate, including calcium phosphate dihydrate (brushite), uric acid, magnesium ammonium phosphate (struvite) and cystine. In special cases, other types of urinary stones arise: xanthine

\footnotetext{
Correspondence address:

Monika Stępień, Department of Radiology, Jagiellonian University in Kraków, Collegium Medicum, 19 Kopernika St., 31-501 Kraków, Poland,

e-mail:mdmonikas@gmail.com
}

Authors' contribution:

A Study design · B Data collection · C Statistical analysis · D Data interpretation · E Manuscript preparation · F Literature search · G Funds collection 
deposits (in inherited deficiency of xanthine oxidase), acid ammonium urate (in chronic diarrhea and hypokalemia), oxypurinol and xanthine (in chronic treatment with allopurinol or triamterene) [2].

Treatment of urolithiasis depends on the chemical composition, location and size of the deposits and may be conservative or surgical. Knowledge of the chemical composition may be crucial in choosing the right therapy and in forecasting the treatment results.

Chemolysis of deposits is possible only in some types of nephrolithiasis.

In the case of struvite, brushite, cystine and uric acid deposits, percutaneous rinsing with solubilizing agents (hemiacridrin, Suby's G, THAM, N-acetylcysteine) may be used.

For uric acid deposits, oral medications may also be effective to alkalinize the urine to $\mathrm{pH}$ of 6.5-7.2 using alkaline citrates or sodium bicarbonate; invasive treatment is implemented only when conservative treatment is ineffective.

Cystine deposits are best dissolved at $\mathrm{pH}=7.0-7.5$ using mainly D-penicillamine, alpha-MPG, captopril and tiopronin [3].

The most common method of urolithiasis treatment is lithotripsy with shock waves generated extracorporeally (extracorporeal shock wave lithotripsy - ESWL). This method uses electromagnetic, electrohydraulic or piezoelectric lithotripters, locating the stone with fluoroscopy and/or ultrasound. The optimal location for ESWL is the pelvis and calyces (especially upper and middle) and the proximal part of the ureter (above the sacroiliac joint line); in the distal part of the ureter, it is better to perform ureteroscopy. Usually ESWL is used in kidney deposits up to $20 \mathrm{~mm}$ in size and ureter deposits up to $10 \mathrm{~mm} \mathrm{[3].} \mathrm{Calci-}$ um oxalate and uric acid stones disintegrate relatively easily. Calcium phosphates, especially those containing brushite, crumble worse and cystine deposits definitely badly [4].

Other methods of treatment include: ureterorenoscopy (URS) involving removal of ureter stones under visual control using a ureterorenoscope and percutaneous nephrolithotomy (PCNL) based on removal of the deposit from the kidney or upper ureter, whole or crushed, using an endoscope inserted directly into the pelvico-calyceal system.

Determination of the chemical composition of ureteral concrements in vivo could significantly influence the selection of optimal therapeutic treatment.

Until recently, we did not have a diagnostic method to obtain such data before spontaneous expelling or surgical removal of concrement.

This situation changed only after the introduction of dual energy computed tomography (DECT).

The aim of DECT is to obtain two sets of data (images) during the acquisition of the same anatomical area at different values of X-ray energy (usually $80 \mathrm{keV}$ and $140 \mathrm{keV}$ ) [5].

The theoretical principles of DECT were developed in the 1970s, but the first computed tomography scanners with the practical implementation of this method, using various technical solutions, appeared only a few years ago.

DECT allows deposits containing uric acid to be distinguished from deposits which do not contain uric acid, in tests performed both in vivo and in vitro [6]. Due to the different chemical structure, uric acid deposits show higher radiodensity Hounsfield unit values using high-energy radiation (at higher kilovoltage), while other types of deposits (including calcium deposits) show higher radiodensity Hounsfield unit values using low-energy radiation (low kilovoltage) [7]. Data obtained in DECT are postprocessed using dedicated software, in which voxels containing uric acid are coded in a different color compared to voxels that do not contain uric acid.

The assessment of the usefulness of DECT in determining the chemical composition of urinary stones is an increasingly common subject of research [7-11]. Most of them are in vivo studies, but some focus on in vitro evaluation.

The aim of our study was to evaluate the possibilities of in vitro DECT assessment of urinary stones' chemical composition.

\section{Material and methods}

Six samples of surgically removed urinary stones were analyzed. The individual samples were placed in closed plastic containers. There were both single large and numerous small concrements within the samples.

The samples were scanned in the DECT technique using a Toshiba Aquilion Prime 80 computed tomography scanner, with the following parameters: voltage and current $135 \mathrm{kV}$ and $100 \mathrm{~mA}$ or $80 \mathrm{kV}$ and $570 \mathrm{~mA}$, rotation time $0.5 \mathrm{~s}$, configuration of detectors $80 \times 0.5 \mathrm{~mm}$, pitch 0.171 , reconstruction interval and slice thickness $1 \mathrm{~mm}$, FOV (field of view) $65 \mathrm{~mm}$.

The CTDIvol (CT dose index) for the above protocol was $33.2 \mathrm{mGy}$.

After processing the data from scanning in the DECT technique, the axial images and the secondary $2 \mathrm{D}$ and $3 \mathrm{D}$ reconstructions were obtained.

The analysis of the above images was performed using Toshiba DE Stone Analysis software.

The elements of samples containing uric acid were marked by the program in red, while those containing calcium compounds were marked in white (Figures 1 and 2).

The composition of stone samples determined in the above way was compared with their composition evaluated in the laboratory tests during physico-chemical analysis.

\section{Results}

After DECT examination of six samples and data processing, axial images and secondary $2 \mathrm{D}$ and $3 \mathrm{D}$ reconstructions were obtained, with elements containing uric acid marked in red, and those containing calcium compounds in white (Figures 1-8). 


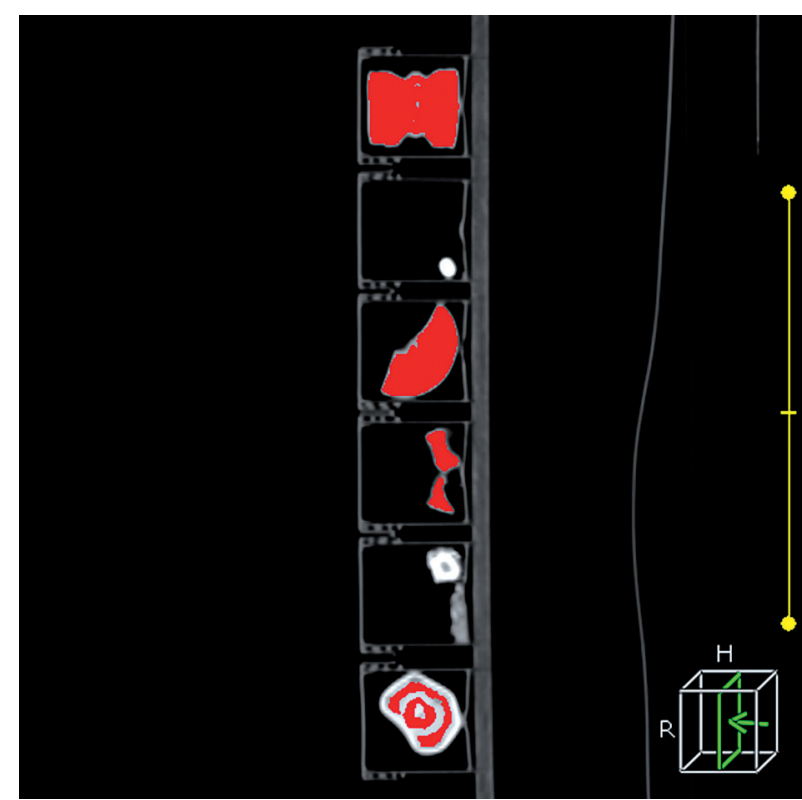

Figure 1. DECT -6 samples of surgically removed urinary stones $-2 \mathrm{D}$ reconstruction in the sagittal plane; respectively from above: uric acid, calcium oxalate, uric acid, uric acid, calcium phosphate, layered stone containing both uric acid and calcium compounds; uric acid marked in red, calcium compounds in white

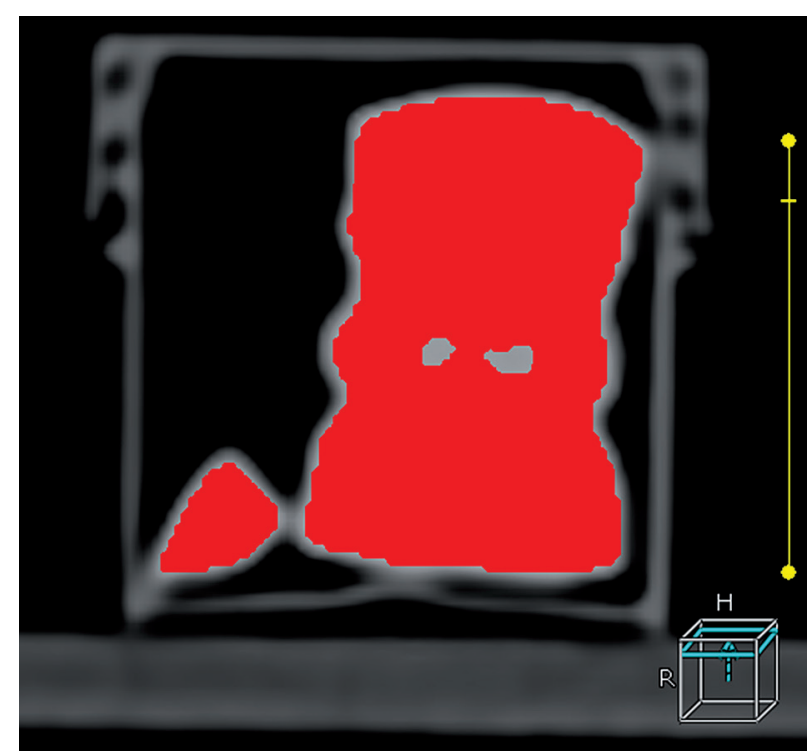

Figure 3. DECT - a sample of surgically removed urinary stone - axial cross section - uric acid

In five out of six samples (Figures 3-7) the assessment of the stones' composition in DECT (three samples determined as uric acid and two samples as calcium compounds) agreed with the result of physico-chemical analysis (three samples defined as uric acid, one as calcium phosphate, one as calcium oxalate). In DECT technique, it was not possible to determine more precisely the type of calcium compounds (calcium phosphate vs. calcium oxalate), as verified in the physico-chemical study.

In one of the six samples (Figure 8), the stone determined in the physico-chemical analysis as a uric acid de-

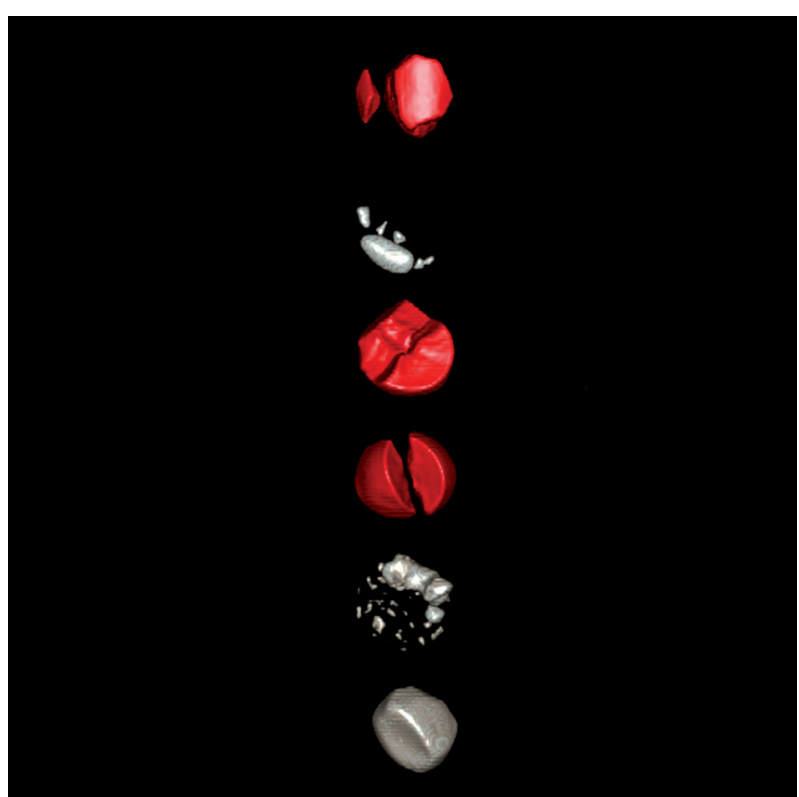

Figure 2. DECT -6 samples of surgically removed urinary stones $-3 \mathrm{D}$ reconstruction; respectively from above: uric acid, calcium oxalate, uric acid, uric acid, calcium phosphate, layered stone containing both uric acid and calcium compounds; uric acid marked in red, calcium compounds in white

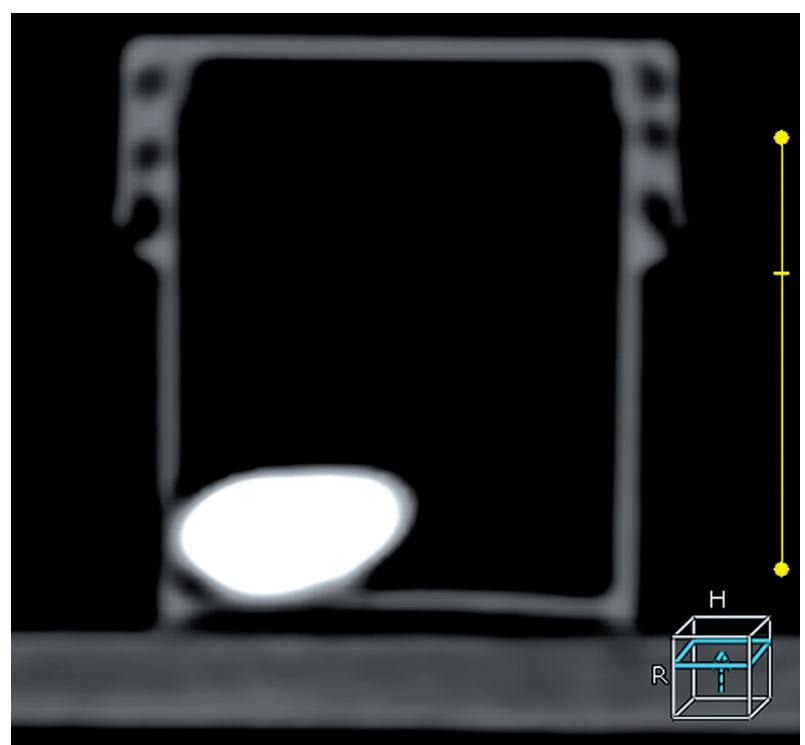

Figure 4. DECT - a sample of surgically removed urinary stone - axial cross section - calcium oxalate

posit, in DECT turned out to have a complex layered structure, containing both uric acid and calcium compounds.

\section{Discussion}

Commonly available multi-row CT allows the assessment of only the size and location of stones. Due to the overlapping ranges of $\mathrm{CT}$ attenuation values among different types of calculi, it is impossible to determine their chemical composition in a typical CT scan [12].

The introduction of the DECT method changed it dual energy computed tomography allows one to differ- 


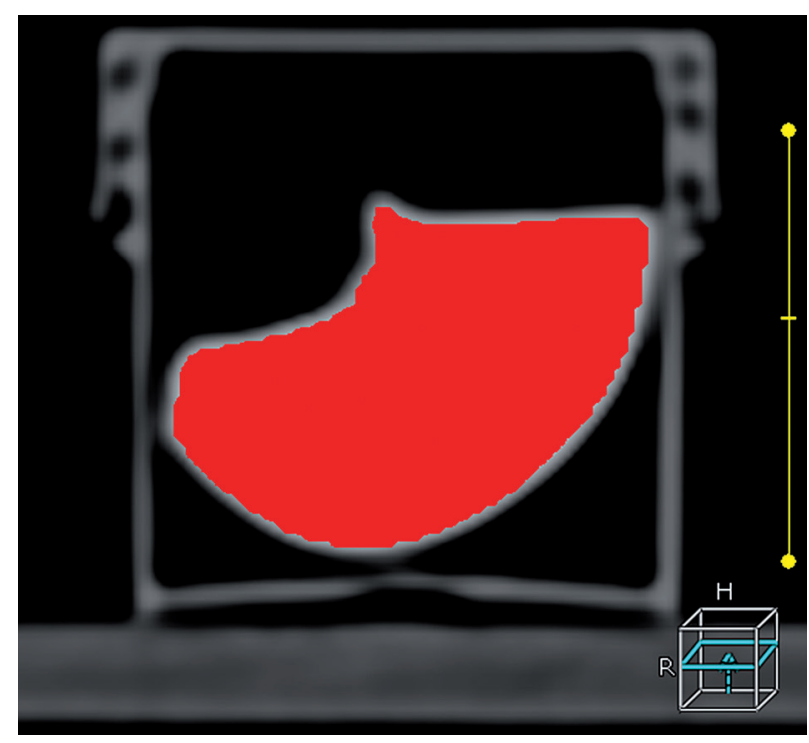

Figure 5. DECT - a sample of surgically removed urinary stone - axial cross section - uric acid

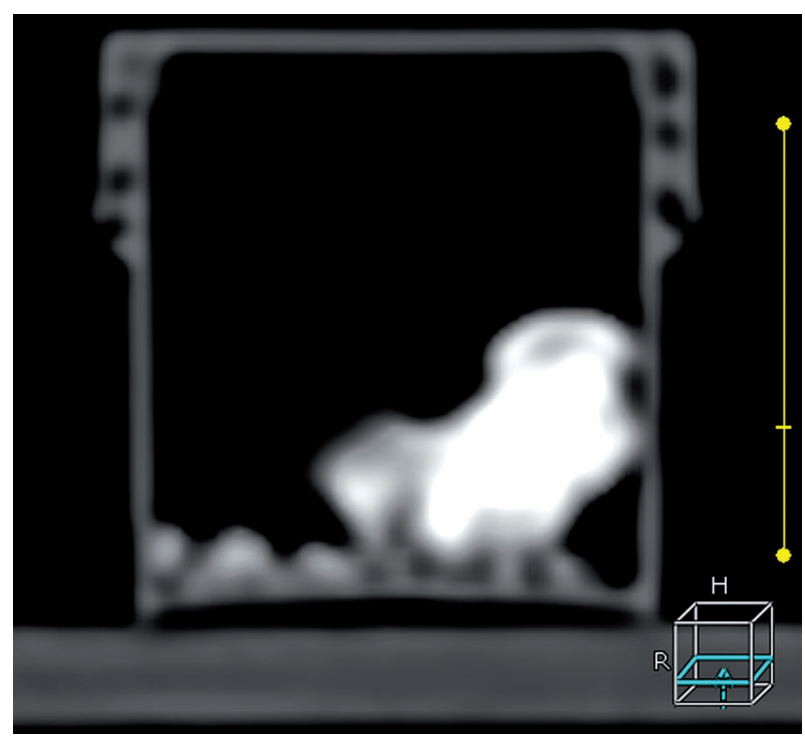

Figure 7. DECT - a sample of surgically removed urinary stone - axial cross section - calcium phosphate

entiate with high accuracy uric acid from non-uric acid stones, both in vivo [7,8] and in vitro [9-11].

Our in vitro DECT study of the stones' composition, with analysis performed by the scanner manufacturer's software, confirmed the possibility of distinguishing uric acid from calcium-containing concrements; however, it was not possible to determine more precisely the type of calcium compounds.

Similarly, Graser et al. [9] found that in DECT it is possible to reliably distinguish between uric acid and other types of stones, but the range of attenuation values for struvite stones largely overlapped with the range for mixed stones and partially with the range for calcified concrements.

According to data from the literature, more groups of deposits can be distinguished by means of more advanced software and modification of the beam filtration.

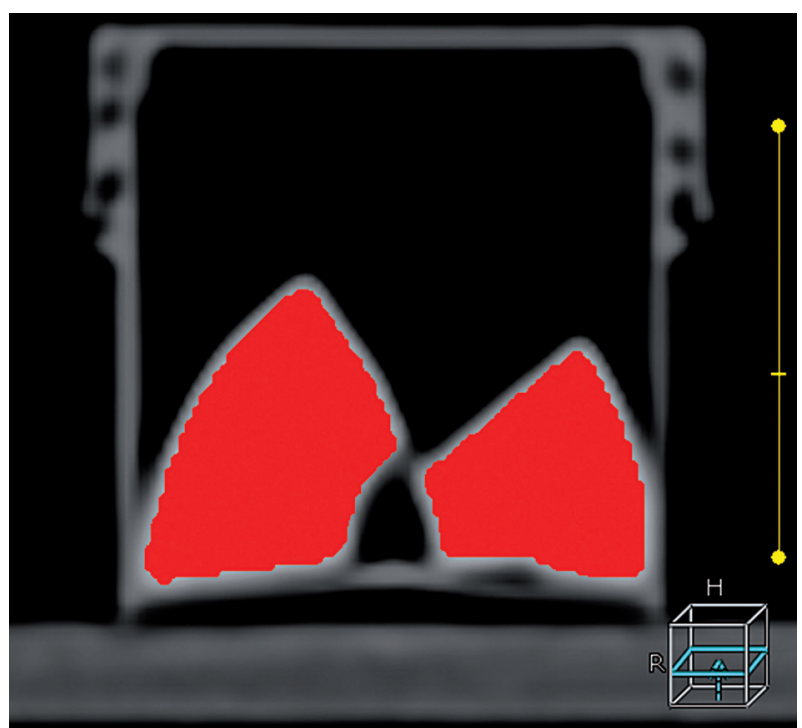

Figure 6. DECT - a sample of surgically removed urinary stone - axial cross section - uric acid

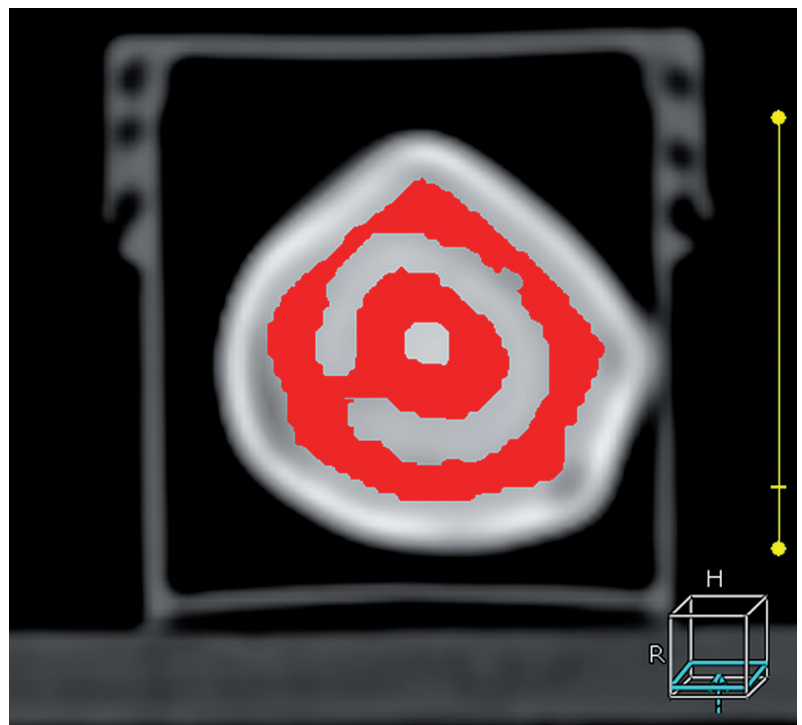

Figure 8. DECT - a sample of surgically removed urinary stone - axial cross section - layered concrement containing both uric acid and calcium compounds

Hidas et al. [8], in a study using a CT scanner with a single $\mathrm{x}$-ray tube and a dual array of high and low energy photon detectors, assessed the urinary stones in patients in vivo preoperatively and compared the results with postoperative X-ray diffraction analysis of the stones.

Before in vivo CT scanning, the researchers created a catalog of stones with known chemical composition, in which they assigned deposits to seven groups (including uric acid, cystine, struvite, apatite, weddellite, whewellite and brushite) and then they evaluated the ratio of low-energy attenuation to high-energy attenuation for every above-mentioned stone. Using data obtained from an in vitro CT study, researchers were able to distinguish between uric acid, cystine and calcium stones, in a CT study performed in vivo. Due to some similarity of chemical structure, technical limitations of the CT scanner 
and differences in radiation beam absorption in patients with different body structures, it was not possible to distinguish between struvite stones and subtypes of calcium deposits.

Fung et al. [10], using a dual-source CT scanner with a tin filter attached to the X-ray tube emitting high-energy radiation, were able to differentiate in vitro stones containing uric acid, calcium oxalates and calcium phosphates. Application of a tin filter resulted in better separation of the attenuation values of oxalates and calcium phosphates, enabling their differentiation.

Similarly, Qu et al. [11] evaluated the composition of stones in vitro, using a dual energy, dual-source CT scanner with an additional tin filter. In this study it was possible to distinguish five groups of concrements (group 1: uric acid, uric acid dihydrate, ammonium acid urate; group 2: cystine; group 3: struvite; group 4: calcium oxalate monohydrate, calcium oxalate dihydrate, brushite; group 5: hydroxyapatite and carbonate apatite). However, the authors noted partial overlap of the features of some concrement types. Moreover, the additional tin filter is an option available only from some manufacturers.

In our dual energy CT study, in most cases the assessment of the chemical composition of stones agreed with the result of the physico-chemical analysis (uric acid vs. calcium deposits); in one case, however, we found discordance. The reason was a mixed-layered structure of the stone, built of both uric acid and calcium compounds.

Similarly, Manglaviti et al. [7], investigating the concrements with a dual-source CT apparatus, in four cases found a discrepancy between the CT assessment of the chemical composition of concrements and the result of the crystallographic study. All the erroneously evaluated concrements had a mixed chemical structure (they con- tained uric acid and hydroxyapatite) and had a diameter below $1 \mathrm{~cm}$. Probably the mixed chemical structure of the concrement, combined with its small size, contribute to the accuracy reduction in the DECT technique analysis.

Our study concerned the use of DECT technique to assess urinary stone composition in vitro, whereas in the case of such in vivo assessment, the radiation dose used during the study becomes an important factor. The computed tomography dose index (CTDIvol) for our protocol was $33.2 \mathrm{mGy}$, which is a significantly higher value than that used in standard abdominal and pelvic examinations (about 7-18 mGy) and much higher than the value in the dedicated low-dose protocol for the assessment of urinary tract stones (approx. $4 \mathrm{mGy}$ ). Fortunately, in the case of in vivo evaluation, the DECT protocol with the higher dose is used only in the range including the location of the concrements. Moreover, Chaytor et al. [13] found that reliable in vivo DECT assessment of stone composition is possible using a lower CTDIvol value of $11.7 \mathrm{mGy}$.

\section{Conclusions}

1. Dual-energy CT technique enables differentiation of uric acid from calcium-containing urinary stones.

2. Using the available hardware and software, more detailed distinction of calcified stone subtypes was not possible.

3. Evaluation of urinary stones' chemical composition in dual-energy computed tomography may be limited in the case of concrements with mixed chemical composition.

\section{Disclosure}

The authors report no conflict of interests.

\section{References}

1. Edvardsson V, Indridason O, Haraldsson G, et al. Temporal trends in the incidence of kidney stone disease. Kidney Int 2013; 83: 146-152.

2. Sułowicz W, Stompór T, Drabczyk R. Kamica nerkowa. In: Gajewski P (ed.). Interna Szczeklika 2016. Medycyna Praktyczna, Liszki 2016; 1567.

3. Świniarski P. Kamica nerkowa - rodzaje, objawy, leczenie. Przegląd Urologiczny 2014; 2: 13.

4. Koźmińska E. Kamica moczowa. In: Borkowski A (ed.). Urologia. Wydawnictwo Lekarskie PZWL, Warszawa 2015; 207-210.

5. Johnson TRC. Dual-energy CT: general principles. AJR Am J Roentgenol 2012; 199: S3-S8.

6. Dale J, Gupta Rajan T, Marin D, et al. Imaging advances in urolithiasis. J Endourol 2017; 31: 623-629.

7. Manglaviti G, Tresoldi S, Guerrer CS, et al. In vivo evaluation of the chemical composition of urinary stones using dual-energy CT. AJR Am J Roentgenol 2011; 197: W76-83.

8. Hidas G, Eliahou R, Duvdevani M, et al. Determination of renal stone composition with dual-energy CT: in vivo analysis and comparison with x-ray diffraction. Radiology 2010; 257: 394-401.

9. Graser A, Johnson TR, Bader M, et al. Dual energy CT characterization of urinary calculi: initial in vitro and clinical experience. Invest Radiol 2008; 43: 112-119.

10. Fung GS, Kawamoto S, Matlaga BR, et al. Differentiation of kidney stones using dual-energy CT with and without a tin filter. AJR Am J Roentgenol 2012; 198: 1380-1386.

11. Qu M, Ramirez-Giraldo JC, Leng S, et al. Dual-energy dual-source CT with additional spectral filtration can improve the differentiation of non-uric acid renal stones: an ex vivo phantom study. AJR Am J Roentgenol 2011; 196: 1279-1287.

12. Platt JF, Kaza RK. Renal applications of dual-energy CT. Abdom Radiol (NY) 2016; 41: 1122-1132.

13. Chaytor RJ, Rajbabu K, Jones PA, et al. Determining the composition of urinary tract calculi using stone-targeted dual-energy CT: evaluation of a low-dose scanning protocol in a clinical environment. Br J Radiol 2016; 89: 20160408. 